\title{
Valence-band orbital character of CdO: A synchrotron-radiation photoelectron spectroscopy and density functional theory study
}

\author{
J. J. Mudd,${ }^{1, *}$ Tien-Lin Lee, ${ }^{2}$ V. Muñoz-Sanjosé, ${ }^{3}$ J. Zúñiga-Pérez, ${ }^{4}$ D. J. Payne,${ }^{5}$ R. G. Egdell, ${ }^{6}$ and C. F. McConville ${ }^{1, \dagger}$ \\ ${ }^{1}$ Department of Physics, University of Warwick, Coventry, CV4 7AL, United Kingdom \\ ${ }^{2}$ Diamond Light Source Ltd., Harwell Science and Innovation Campus, Didcot, OX11 ODE, United Kingdom \\ ${ }^{3}$ Departamento de Fisica Aplicada y Electromagnetismo, Universitat de Valéncia, C/Dr. Moliner 50, 46100 Burjassot, Spain \\ ${ }^{4}$ Centre de Recherche sur lHétéro-Epitaxie et ses Applications, Centre National de la Recherche Scientifique, Parc de Sophia Antipolis, \\ Rue Bernard Grégory, 06560 Valbonne, France \\ ${ }^{5}$ Department of Materials, Imperial College London, London, SW7 2AZ, United Kingdom \\ ${ }^{6}$ Inorganic Chemistry Laboratory, Department of Chemistry, University of Oxford, South Parks Road, Oxford OX1 3QR, United Kingdom
}

(Received 4 February 2014; revised manuscript received 19 March 2014; published 11 April 2014)

\begin{abstract}
$N$-type $\mathrm{CdO}$ is a transparent conducting oxide (TCO) which has promise in a number of areas including solar cell applications. In order to realize this potential a detailed knowledge of the electronic structure of the material is essential. In particular, standard density functional theory (DFT) methods struggle to accurately predict fundamental material properties such as the band gap. This is largely due to the underestimation of the $\mathrm{Cd} 4 d$ binding energy, which results in a strong hybridization with the valence-band (VB) states. In order to test theoretical approaches, comparisons to experiment need to be made. Here, synchrotron-radiation photoelectron spectroscopy (SR-PES) measurements are presented, and comparison with three theoretical approaches are made. In particular the position of the $\mathrm{Cd} 4 d$ state is measured with hard x-ray PES, and the orbital character of the VB is probed by photon energy dependent measurements. It is found that LDA $+\mathrm{U}$ using a theoretical $U$ value of $2.34 \mathrm{eV}$ is very successful in predicting the position of the $\mathrm{Cd} 4 d$ state. The VB photon energy dependence reveals the $\mathrm{O} 2 p$ photoionization cross section is underestimated at higher photon energies, and that an orbital contribution from Cd $5 p$ is underestimated by all the DFT approaches.
\end{abstract}

DOI: 10.1103/PhysRevB.89.165305

PACS number(s): 68.47.Gh, 71.15.Mb, 79.60.Bm

\section{INTRODUCTION}

$\mathrm{CdO}$ is a rocksalt metal oxide which is amenable to $n$-type doping resulting in high conductivity and optical transparency. It shows promise for device applications due to its ability to achieve high $n$-type carrier concentrations $\left(\sim 10^{21} \mathrm{~cm}^{-3}\right)$ and high electron mobilities $\left(220 \mathrm{~cm}^{2} \mathrm{~V}^{-1} \mathrm{~s}^{-1}\right)$ [1-3]. It is also a promising candidate to alloy with $\mathrm{ZnO}$ to form $\mathrm{Cd}_{x} \mathrm{Zn}_{1-x} \mathrm{O}$, potentially achieving devices operating across the visible spectrum [4,5]. However, in order to exploit these potential applications a detailed knowledge of the electronic structure of both $\mathrm{CdO}$ and $\mathrm{ZnO}$ is required [6,7]. In particular, predicting the band gap of these materials is crucial for most applications. However, many current theoretical approaches predict $\mathrm{CdO}$ to be a semimetal [8]. This is a result of the underestimation of the binding energy of the $\mathrm{Cd} 4 d$ states, which results in strong $p$ - $d$ hybridization in the valence band (VB) and a reduction of the band gap. There have been several theoretical attempts to correct this problem including the use of hybrid functionals $[9,10]$ and $\mathrm{GW}$ quasiparticle calculations $[11,12]$. These approaches have had some success in predicting the location of the shallow $\mathrm{Cd} 4 d$ states and the band gap; however, they are also computationally more complex and therefore less widely applied. An alternative approach is LDA + U [13-15], where an on-site, orbital dependent Coulomb term is added to the local density approximation (LDA) potential. This allows the position of the $\mathrm{Cd} 4 d$ states to be corrected and should therefore improve the associated theoretical predictions.

\footnotetext{
*j.j.mudd@warwick.ac.uk

†c.f.mcconville@warwick.ac.uk
}

In order to assess the merits of these theoretical approaches, comparisons with experimental data need to be made. Photoemission is an ideal technique for this as it essentially allows the VB density of states (DOS) to be observed. To the best of our knowledge there have been two previous publications comparing theoretical predictions to experimental photoemission data for $\mathrm{CdO}$, King et al. [16] and Dou et al. [17]. This work significantly extends both of these comparisons, by both the use of additional theoretical approaches and synchrotron-radiation techniques.

Synchrotron-radiation excited photoelectron spectroscopy (SR-PES) has been used to obtain both hard x-ray photoemission (HAXPES) data of the $\mathrm{CdO} \mathrm{VB}$ and $\mathrm{Cd} 4 d$ regions for comparison to density functional theory (DFT), and a series of $\mathrm{CdO}$ VB spectra over a range of photon energies to allow the VB orbital character to be investigated in more detail. With its higher incident photon energy, HAXPES is a preferred technique when comparing to theoretical calculations as it is more bulk sensitive than traditional photoemission [18], allowing for better comparison with the theoretical bulk calculations. It is also of benefit that photoionization cross sections are smoothly varying at high photon energies, allowing trends to be more easily observed. Theoretically three DFT functionals were chosen (LDA, PBE-GGA, and LDA + U), due to their widespread availability and relative computational simplicity.

\section{EXPERIMENTAL DETAILS}

Epitaxial $\mathrm{CdO}(100)$ single crystal thin films were grown on $r$-plane sapphire substrates using metal organic vapor-phase epitaxy (MOVPE), further details of which can be found 
elsewhere [21]. The sample was prepared in ultra-high vacuum (UHV) by annealing at $550{ }^{\circ} \mathrm{C}$ for $45 \mathrm{~min}$, an approach which has previously been shown to be very effective [16]. Prior to the PES measurements the surface order was confirmed by LEED, which showed a sharp $(1 \times 1)$ diffraction pattern indicating high surface quality. Additionally, no contamination was observed during the PES measurements.

Photoemission spectra were obtained at the I09 beamline of Diamond Light Source, UK. The I09 beamline offers photon energies from $100 \mathrm{eV}$ to $18 \mathrm{keV}$, achieved using two canted undulators, providing both soft and hard $\mathrm{x}$ rays focused to the same point on the sample. Hard $\mathrm{x}$ rays $(h v \geqslant 2500 \mathrm{eV})$ were monochromated using a cooled $\mathrm{Si}(111)$ double crystal monochromator. To maintain sufficient energy resolution at the two highest photon energies an additional Si channel-cut high resolution monochromator, $\mathrm{Si}(444)$ for $h v=7935 \mathrm{eV}$ and $\operatorname{Si}(004)$ for $h v=6054 \mathrm{eV}$, was used. Soft $\mathrm{x}$ rays $(h v=600 \mathrm{eV})$

(a)

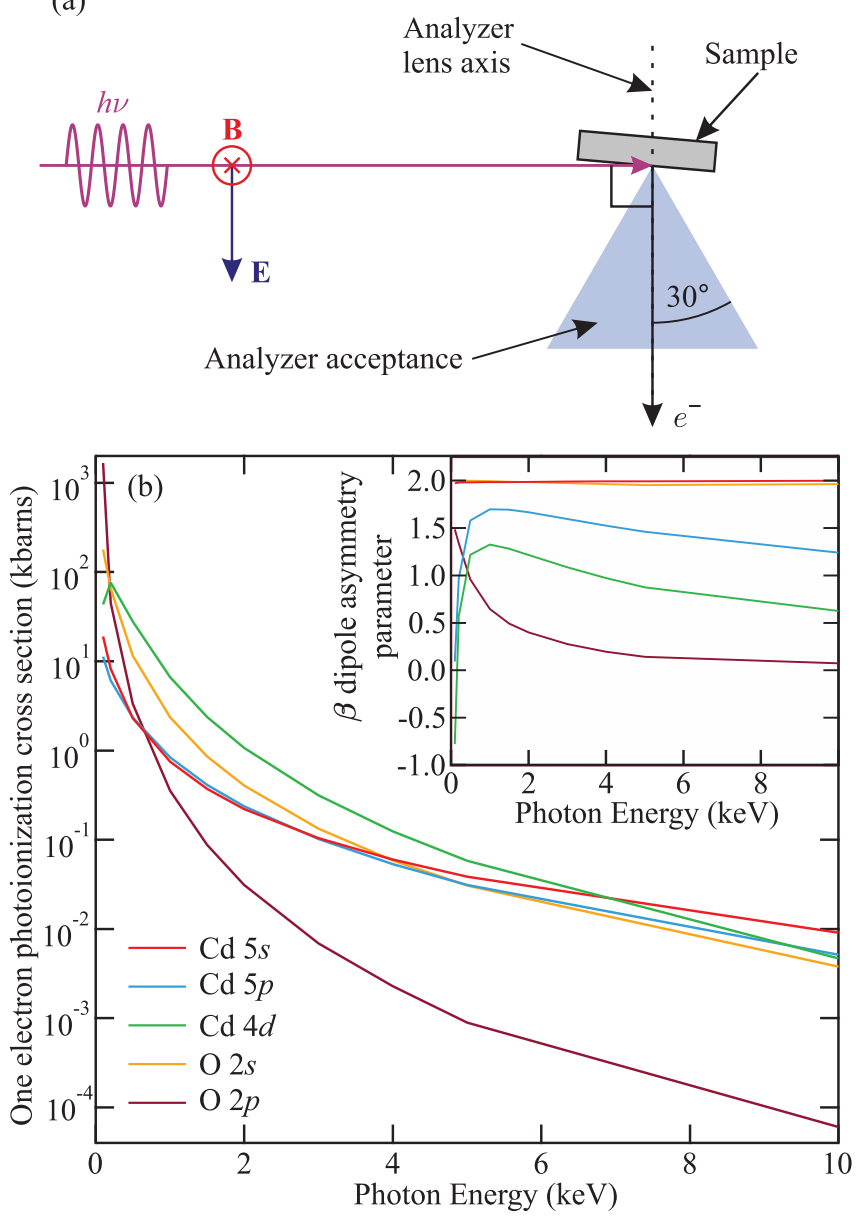

FIG. 1. (Color online) (a) Plan view of the experimental geometry used. The photon beam is perpendicular to the analyzer lens axis and the sample is placed at grazing incidence to the photon beam. The electric polarization vector is coincident with the analyzer lens axis. The $\pm 30^{\circ}$ angular acceptance of the electron analyzer is illustrated by the shaded blue triangle. (b) The one-electron photoionization cross sections and (inset) $\beta$ dipole asymmetry parameters for the orbitals constituting the $\mathrm{CdO}$ valence band. Shown for photon energies 0.1 to $10 \mathrm{keV}$. Note the logarithmic scale. Data from Trzhaskovskaya et al. $[19,20]$. were monochromated using a plane grating monochromator (300 lines $/ \mathrm{mm}$ ). The endstation is equipped with a VG Scienta EW4000 electron analyzer with $\pm 30^{\circ}$ angular acceptance. In the experimental geometry at I09 shown in Fig. 1(a), the photon beam is perpendicular to the electron emission direction. The photon beam is polarized in the plane of the orbit (horizontally) resulting in the electric vector being aligned with the electron emission direction. The sample was placed in a grazing incidence geometry $\left(\sim 5^{\circ}\right)$, with the surface normal in the plane defined by the photon beam and electron emission direction ( $p$-polarized), thereby significantly enhancing the count rate. The experiments were performed at room temperature. The total experimental energy resolution is $<0.6 \mathrm{eV}$, for all photon energies. All experimental data are shown with energy scales referenced to the valence-band maximum (VBM) to allow for comparison to the theoretical calculations.

\section{COMPUTATIONAL DETAILS}

\section{A. Density functional theory}

DFT calculations were performed using the full potential linearized augmented plane wave (FP-LAPW) method with local orbitals [22], as implemented in the ELK open source code [23]. Calculations were performed on $\mathrm{CdO}$ in the rocksalt structure. Three different DFT functionals were applied; Perdew-Zunger local density approximation (LDA) [24], Perdew-Burke-Ernzerhof generalized gradient approximation (PBE-GGA) [25], and the LDA + U approach within the fully localized limit (FLL) [13-15]. The difficulty in applying this method is choosing a suitable value of U. Here a theoretically calculated value of $U=2.34 \mathrm{eV}$ suggested by Janotti et al. [26] is used, applied to the $\mathrm{Cd} 4 d$ states. Structural optimization was conducted with each functional by performing calculations over a range of unit cell volumes, and fitting the energy-volume curve with the Murnaghan equation of state [27]. From this the equilibrium lattice parameter was obtained, and this was used when calculating the band structures and partial density of states (PDOS). Convergence tests with respect to the plane wave cutoff and $k$-point mesh indicate the results are numerically converged to $\sim 5 \mathrm{meV}$. The wave functions inside the muffin tins were expanded up to $l_{\max }=8$ and the plane waves were expanded with a cutoff of $R_{\min }^{\mathrm{MT}} \times K_{\max }=8.5$, where $R_{\text {min }}^{\mathrm{MT}}$ is the smallest muffin tin radius and $K_{\max }=|\mathbf{G}+\mathbf{k}|$ the maximum value of the wave vector. The muffin-tin radii were chosen as $R_{\mathrm{Cd}}^{\mathrm{MT}}=1.35 \AA$ and $R_{\mathrm{O}}^{\mathrm{MT}}=0.94 \AA$. A $k$ mesh of $8 \times 8 \times 8$ was found to be sufficient for all calculations.

\section{B. Experimental comparison}

DFT has been used to calculate the orbitally resolved PDOS. To allow comparison with experimental data the PDOS was multiplied by the one-electron photoionization cross sections, including an asymmetry correction. To obtain these one-electron photoionization cross sections, the tabulated values of the subshell photoionization cross sections, calculated by Trzhaskovskaya et al. [19,20], were summed and divided by the orbital occupancy of a free atom. As the $\mathrm{Cd} 5 p$ shell is unoccupied in a $\mathrm{Cd}$ atom, the cross section was estimated by applying the In $5 p / \operatorname{In} 5 s$ ratio to the Cd $5 s$ cross section. The 
Cd $5 p$ dipole asymmetry parameter was assumed to be equal to the In $5 p$ dipole asymmetry parameter, a valid approach as the dipole asymmetry changes only weakly with atomic number. The resulting one-electron photoionization cross sections and $\beta$ dipole asymmetry parameters are shown in Fig. 1(b).

The differential cross section including the dipolar and nondipolar asymmetry parameters, for linearly polarized light, is given by [30]

$$
\frac{d \sigma_{i}}{d \Omega}=\frac{\sigma_{i}}{4 \pi}[1+\underbrace{\beta P_{2}(\cos \theta)}_{\text {dipolar }}+\underbrace{\left(\delta+\gamma \cos ^{2} \theta\right) \sin \theta \cos \phi}_{\text {nondipolar }}],
$$

where $P_{2}$ is the Legendre polynomial, $\sigma_{i}$ is the photoionization cross section, $\beta$ is the dipole asymmetry parameter, and $\gamma$ and $\delta$ are nondipolar asymmetry parameters. The angle $\theta$ is defined between the polarization vector and the electron emission direction, and $\phi$ is defined as the angle between the photon propagation direction and the plane defined by both the electron emission direction and polarization vector, which in the experimental geometry used is always zero. As the nondipolar term displays odd parity, integrating over a symmetric $\theta$ range will result in no contribution from this term, and therefore no dependence on the nondipolar asymmetry parameters. Considering the $\pm 30^{\circ}$ angular acceptance of the electron analyzer and performing the integration of Eq. (1), the total cross section can be written as

$$
\sigma_{i}^{\text {total }}=\frac{\sigma_{i}}{96 \pi}[8 \pi+\beta(9 \sqrt{3}+2 \pi)] .
$$

This expression is used to calculate the total cross section to be applied to each orbital when weighting the PDOS. The photoionization cross section weighted PDOS were then summed and convolved with a $0.7 \mathrm{eV}$ FWHM Gaussian to account for lifetime and phonon broadening, and the finite experimental resolution.

\section{RESULTS AND DISCUSSION}

\section{A. Computational results}

The key numerical results are shown in Table I, which also includes values from recently published hybrid functional (HSE 06) [9], and GW quasiparticle calculations [11]. The calculated lattice parameters agree well with experiment and

TABLE I. Key numerical results from experiment and DFT. The values shown are lattice parameter $a$ and direct $\left(E_{G}^{\mathrm{dir}}\right)$ and indirect band gap $\left(E_{G}^{\text {ind }}\right)$ (negative values indicate semimetallic). The valenceband width $(\Delta \mathrm{VB})$; location of the $\mathrm{Cd} 4 d$ states with respect to the VBM. All energies are in $\mathrm{eV}$.

\begin{tabular}{lllccc}
\hline \hline & $a(\AA)$ & $E_{G}^{\text {dir }}$ & $E_{G}^{\text {ind }}$ & $\Delta \mathrm{VB}$ & $4 d$ states \\
\hline Expt. & $4.695[21]$ & $2.18[28]$ & $\sim 0.9[29]$ & $\sim 4.8$ & $\sim-8.70$ \\
LDA & 4.639 & 1.12 & -0.45 & 4.72 & -6.76 \\
PBE & 4.775 & 0.68 & -0.51 & 3.98 & -6.58 \\
LDA + U & 4.619 & 1.34 & 0.21 & 4.18 & -8.60 \\
HSE 06 & 4.720 & 2.18 & 0.89 & 4.45 & -7.4 \\
GW $^{\mathrm{b}}$ & 4.650 & 2.88 & 1.68 & $\sim 4.75$ & $\sim-9$ \\
\hline \hline
\end{tabular}

${ }^{\mathrm{a}}$ Hybrid functional calculation, Burbano et al. [9].

${ }^{\mathrm{b}} \mathrm{GW}$ quasiparticle calculation, Dixit et al. [11]. previous theoretical calculations $[4,9,11,21]$. The calculated DFT band structures are shown in Fig. 2, where clear differences between the functionals are visible. Both LDA and $\mathrm{PBE}$ predict $\mathrm{CdO}$ to be a semimetal, i.e., the conduction band minimum is below the VBM. This is clearly not consistent with experimental evidence, with the most recent optical measurements placing the direct band gap at $2.18 \mathrm{eV}$ at room temperature [28] and XANES reporting an indirect gap of $\sim 0.9 \mathrm{eV}$ [29]. PBE predicts the location of the $\mathrm{Cd} 4 d$ states to be very similar to LDA but with a slightly narrower overall width. LDA $+\mathrm{U}$ shifts the $\mathrm{Cd} 4 d$ states down by $\sim 2 \mathrm{eV}$. This opens a direct band gap of $1.34 \mathrm{eV}$. Although semiconducting behavior with a nonvanishing indirect gap is now predicted, both the direct and indirect gaps are still too small when compared to experiment. It is worth noting that more sophisticated computational methods such as hybrid functionals [9] can potentially predict the band gap much more accurately.

The partial density of states (PDOS) for each of the functionals is shown in Figs. 3(a)-3(c). It is of note that, for all the functionals, the VB DOS is dominated by $\mathrm{O} 2 p$ character, and the shape of this feature is very similar. PBE predicts a narrower VB than either LDA or LDA $+\mathrm{U}$. The most significant difference is in the orbital character of peak I. LDA predicts this peak to have significant $\mathrm{Cd} 4 d$ character resulting from the underestimation of the $\mathrm{Cd} 4 d$ states binding energy. PBE shows the $\mathrm{Cd} 4 d$ character of this peak to be much reduced giving roughly similar importance to $\mathrm{Cd} 4 d$ and $\mathrm{Cd} 5 s$. For LDA $+\mathrm{U}$ the $\mathrm{Cd} 4 d$ character of peak I is further reduced due to the $\mathrm{Cd} 4 d$ states being shifted to lower energy reducing hybridization. This results in $\mathrm{Cd} 5 s$ contributing more to the DOS in peak I than $\mathrm{Cd} 4 d$. The $\mathrm{Cd}$ $5 p$ and $\mathrm{O} 2 s$ components are also shown. These are often excluded due to their small contributions. However, at higher photon energies their importance to VB-PES increases as their photoionization cross section drops off much less rapidly than O $2 p$ as shown in Figs. 1 and 5.

\section{B. Experimental comparison}

A comparison between the three DFT functionals and experimental valence-band photoemission spectroscopy (VBPES) measurements taken with a photon energy of $6054 \mathrm{eV}$ is shown in Figs. 3(d)-3(i). The higher photon energy compared to earlier studies [16,17] decreases the surface sensitivity, which is beneficial in the case of $\mathrm{CdO}$ as it is known to exhibit surface electron accumulation [31,32], which is not representative of the bulk electronic properties. A Shirley background [33] has been subtracted across the whole region. The PDOS shown in Figs. 3(a)-3(c) have been weighted using the photoionization cross sections and asymmetry corrections appropriate for a photon energy of $6054 \mathrm{eV}$ and then summed, as shown by the shaded gray region. The sum of weighted PDOS was then convolved with a $0.7 \mathrm{eV}$ FWHM Gaussian, resulting in the red line. The experimental spectra and convolved DFT are normalized to peak II of the VB, and the same normalization was applied to both the VB and $\mathrm{Cd} 4 d$ panels to allow the relative intensities to be observed.

The $\mathrm{Cd} 4 d$ region in panels (d)-(f) of Fig. 3 show experimental data placing the $\mathrm{Cd} 4 d$ state at $\sim-8.70 \mathrm{eV}$, in good 

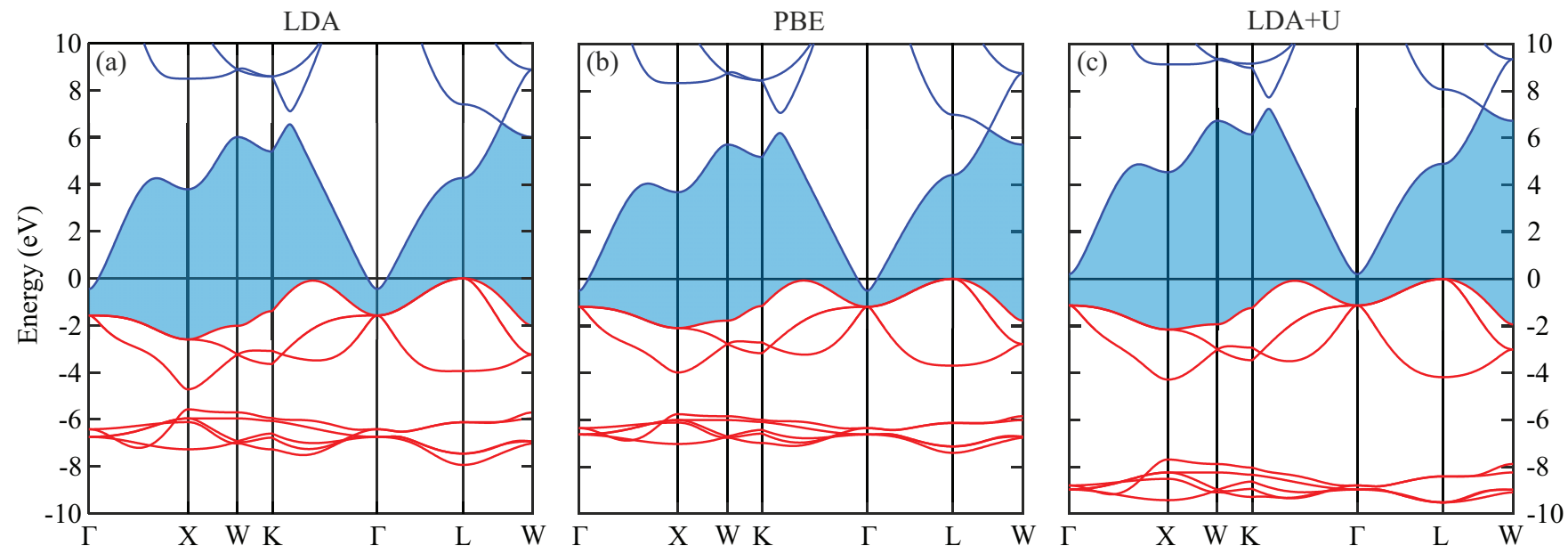

FIG. 2. (Color online) DFT band structures for (a) LDA, (b) PBE, and (c) LDA + U. Blue and red lines denote conduction and valence bands, respectively, the blue shaded region shows the band gap. Note the overlap of the conduction and valence bands predicted by LDA and PBE indicating a semimetallic system. Also note the shift in $\mathrm{Cd} 4 d$ states at around $-8 \mathrm{eV}$ produced by LDA $+\mathrm{U}$.
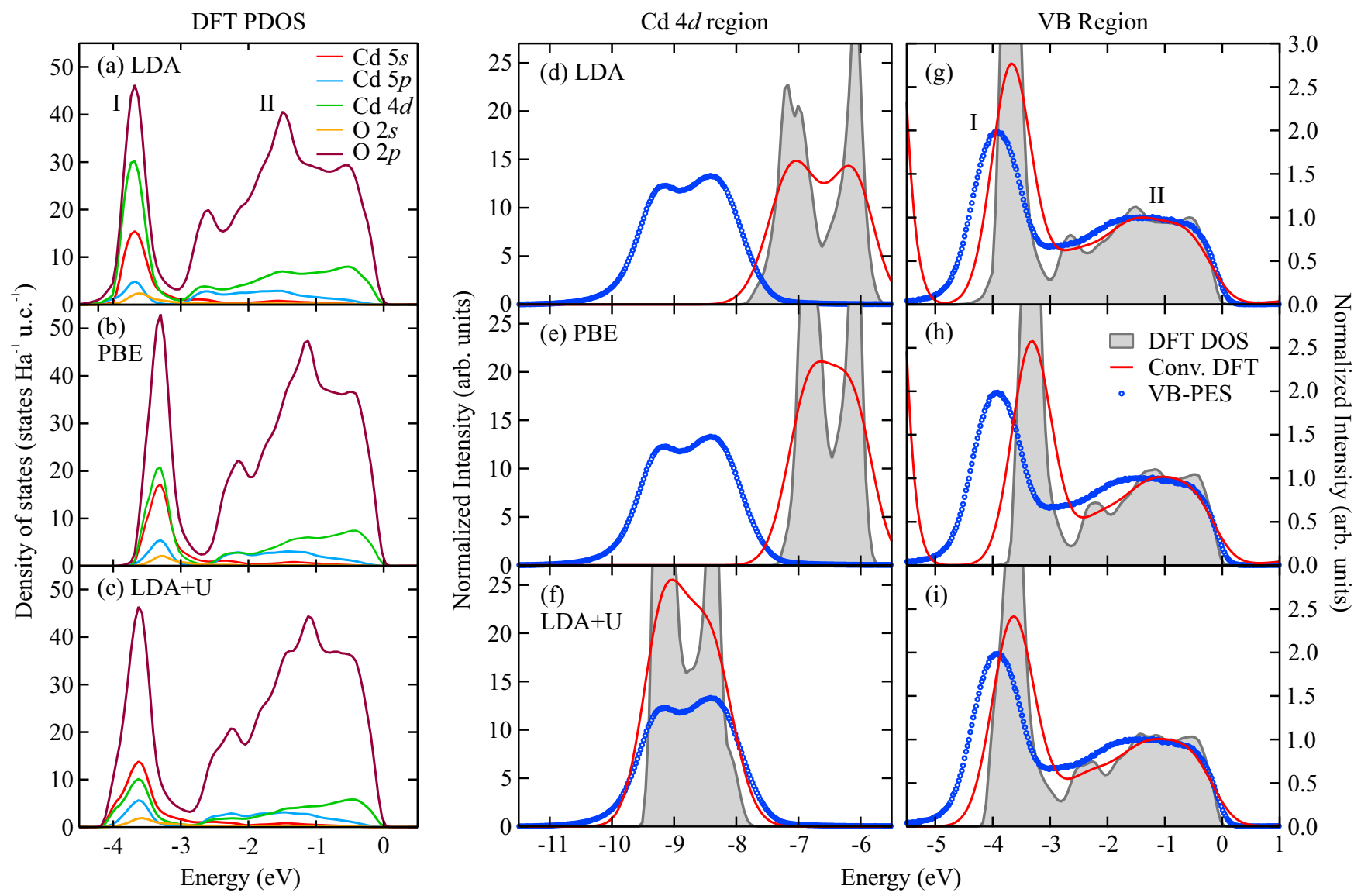

FIG. 3. (Color online) (a)-(c) DFT partial density of states calculated using (a) LDA, (b) PBE, and (c) LDA + U. The peak labels I and II in (a), derived from experimental data, refer to the energy ranges -4.5 to $-3 \mathrm{eV}$ and -3 to $0 \mathrm{eV}$, respectively. (d)-(i) Cd $4 d$ and VB PES spectra compared to DFT, (d) and (g) LDA, (e) and (h) PBE, and (f) and (i) LDA + U. The DFT PDOS are weighted by the photoionization cross sections and summed, shown by the gray shaded areas. The weighted PDOS after convolution with a 0.7 eV FWHM Gaussian are shown by the red line. The experimental data (blue circles) shown is taken with $h v=6054 \mathrm{eV}$, and a Shirley background has been subtracted. All energy scales are referenced to the VBM. The experimental spectra and convolved DFT are normalized to the maximum of VB peak II, and the same normalization has been applied to both the VB and Cd $4 d$ regions; note the different intensity scales. 
agreement with other photoemission measurements [16,17]. Very significant differences between these functionals can be observed, as discussed previously. LDA and PBE place the $\mathrm{Cd} 4 d$ states at approximately the same energy, and this is approximately $2 \mathrm{eV}$ shallower than measured by experiment. LDA $+\mathrm{U}$ corrects for this disagreement extremely successfully, placing the $\mathrm{Cd} 4 d$ states in very good agreement with the experimental data. This can also be compared to recent hybrid functional calculations placing the $\mathrm{Cd} 4 d$ states at $-7.4 \mathrm{eV}$ [9] and to a recent $\mathrm{GW}$ quasiparticle calculation which places the $\mathrm{Cd} 4 d$ states at $\sim-9 \mathrm{eV}$ [11]. It is interesting to note, however, that the shape of the $\mathrm{Cd} 4 d$ states is better reproduced by LDA which shows two resolvable peaks as seen in the experiment. Due to the decreased interaction between the $\mathrm{Cd} 4 d$ states and the VB predicted by LDA $+\mathrm{U}$, and to a lesser extent PBE, the DOS within the $\mathrm{Cd} 4 d$ states is increased. This results in these functionals overestimating the spectral weight of these states relative to the VB.

The VB region shown in panels (g)-(i) of Fig. 3 exhibits a two peak structure which is correctly reproduced by all the DFT functionals. However, it can be seen that all the DFT functionals underestimate the VB width, particularly PBE. This is consistent with previous results [16]. This also means that VB peak I is placed too shallow by around $0.5 \mathrm{eV}$ when compared to the experimental data. The shape of peak II is best reproduced by LDA, which predicts a slightly wider peak than PBE or LDA + U. This is partly due to LDA assigning increased $\mathrm{Cd} 4 d$ character to VB peak II. This is, however, offset by the fact that LDA also places the $\mathrm{Cd} 4 d$ states much too shallow resulting in this effect. The additional $\mathrm{Cd}$ $4 d$ character is also responsible for LDA giving VB peak I more spectral weight than observed in the experimental data. The relative intensities of peaks I and II in the VB region is best reproduced by $\mathrm{LDA}+\mathrm{U}$, which still overestimates peak I intensity.

Figure 4(a) shows VB-PES taken with photon energies ranging from $600 \mathrm{eV}$ to $7935 \mathrm{eV}$. This approach allows the orbital character of VB to be identified by exploiting the change in photoionization cross sections. The spectra have been normalized to the maximum of VB peak II, following a Shirley background subtraction, to allow relative changes to be observed. The key changes in the VB shape observed experimentally are indicated by the two arrows labeled 1 and 2 in Fig. 4(a). Arrow 1 shows that peak I of the VB increases strongly with photon energy relative to peak II. Arrow 2 indicates the region in the center of the VB which also increases relative to peak II, and this appears, like a broadening of peak II, with photon energy. Figures 4(b)-4(d) show the summed DFT PDOS after weighting with different photoionization cross sections as appropriate for each photon energy, and a convolution with a 0.7 eV FWHM Gaussian (as before) to allow direct comparison to the experimental data.

It can be seen qualitatively that the DFT simulations reproduce the trend of peak I increasing in relative intensity with photon energy, but none of the functionals reproduce this behavior quantitatively. The experimental data at the lowest photon energy shows peak I weaker than peak II. DFT doesn't predict any orbitals with lower PDOS in peak I than peak II, indicating the orbital character of the VB predicted by DFT cannot be correct. This result is independent of the photoionization cross sections applied. However, it can be seen that reducing the $\mathrm{Cd} 4 d$ contribution to peak I, which is achieved by LDA $+\mathrm{U}$, does significantly improve the experimental agreement. In the experimental data the relative intensity of peak I increases consistently towards the highest photon energy, whereas the simulations predict an initially rapid increase which slows toward the highest photon energy. To address this photon energy dependence, it seems likely that the photoionization cross sections may exhibit a more complex energy dependence than encapsulated by the one-electron cross sections applied here. The rate of decrease of the $\mathrm{O} 2 p$ one-electron cross section with photon energy appears to be overestimated here, as this would result in the more rapid relative increase in peak I intensity with photon energy, as illustrated by the DFT models. Similar conclusions regarding the $\mathrm{O} 2 p$ photoionization cross sections being underestimated at higher photon energies were reached in the case of $\beta-\mathrm{PbO}_{2}[34,35]$ and $\mathrm{In}_{2} \mathrm{O}_{3}$ [36], where alternative photoionization cross sections calculated by Scofield [37] and by Yeh and Lindau [38] were applied.

A possible explanation is that the very low $\mathrm{O} 2 p$ cross section at higher photon energies is susceptible to enhancement

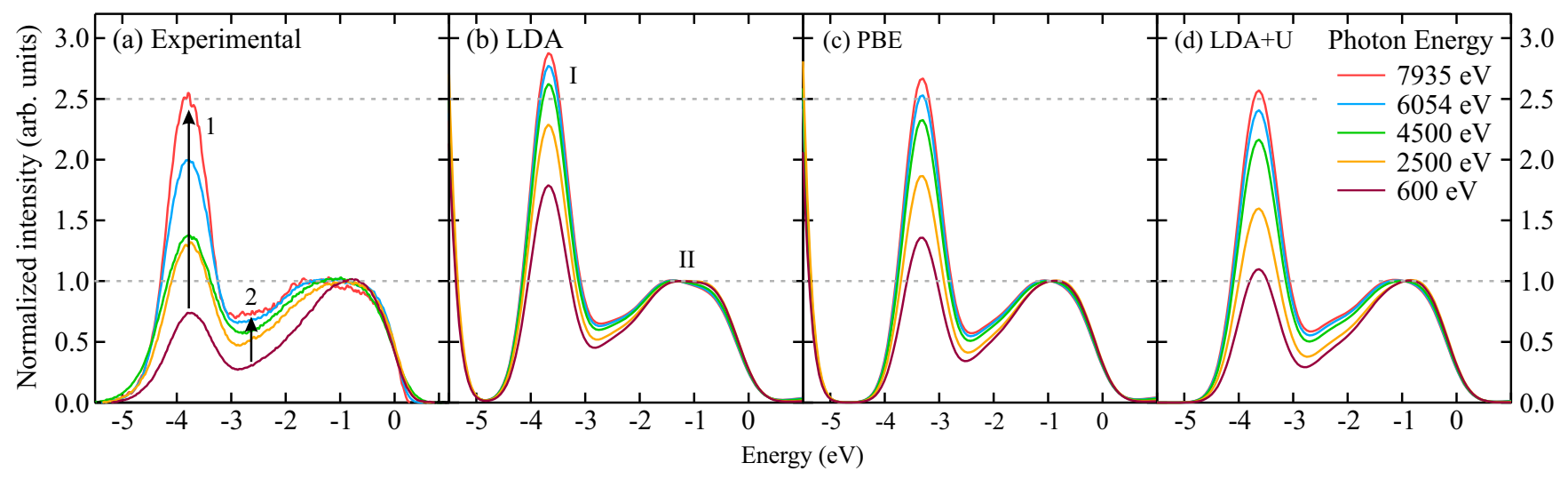

FIG. 4. (Color online) (a) VB-PES taken with photon energies in the range $600 \mathrm{eV}$ to $7935 \mathrm{eV}$; a Shirley background has been subtracted. (b)-(d) Simulations of the VB-PES obtained by applying the photoionization cross sections to the DFT approaches. The arrows numbered 1 and 2 in (a) are guides to the eye, discussed in the text. All spectra have been normalized to the maximum of peak II. The dashed lines are guides to the eye to allow the relative intensities to be observed. 


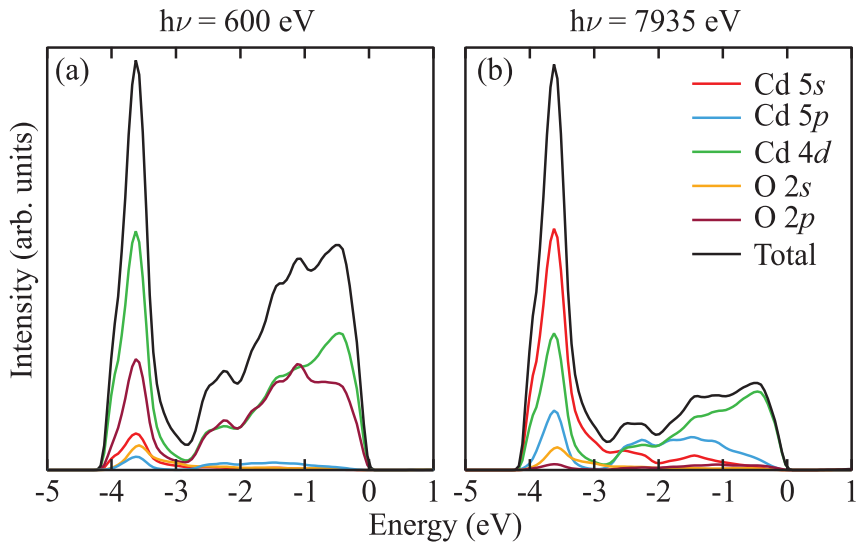

FIG. 5. (Color online) The VB region calculated with LDA + U showing the PDOS weighted by photoionization cross sections for photon energies (a) $600 \mathrm{eV}$ and (b) $7935 \mathrm{eV}$. This allows the effects of the photon energy on the orbital components to be observed.

by interchannel coupling with the much stronger $\mathrm{O} 2 s$ or $\mathrm{O}$ $1 s$ ionizations. Drube et al. [39] have recently shown that interchannel coupling of this sort may be significant well above threshold. In support of this idea we note that the agreement between theory and experiment does significantly improve with increasing photon energy. It is also interesting to note that the intensity ratio of peak I to II is quite well reproduced by all the DFT functionals at the highest photon energy. At this photon energy the VB-PES is dominated by $\mathrm{Cd}$ character, whereas at the lowest photon energy $\mathrm{Cd}$ and $\mathrm{O}$ contribute approximately equally, as shown in Fig. 5. This might suggest that the photoionization cross sections are most accurate within an element and may contribute a larger error when comparing orbital character of two or more elements.

The increasing intensity in the center of the VB with photon energy, shown by arrow 2 at approximately $-2.7 \mathrm{eV}$ in Fig. 4(a), is most probably a result of Cd $5 p$ character in the center of the VB. The $\mathrm{Cd} 5 p$ photoionization cross section decreases much less rapidly than $\mathrm{O} 2 p$ with photon energy, so at higher photon energies its contribution becomes much more significant (see Fig. 5). While all the DFT functionals predict some $\mathrm{Cd} 5 p$ character, it seems likely this contribution is underestimated, as the experimental data shows a much more significant change. Alternatively, the photoionization cross section for $\mathrm{Cd} 5 p$ could be underestimated at higher photon energies. While this is possible due to the extrapolation required to obtain it, it would not be consistent with the trend in peak I which would then show a stronger photon energy dependence if this cross section was increased.

\section{CONCLUSIONS}

The VB electronic structure of $\mathrm{CdO}$ has been investigated with SR-PES, and comparisons to three DFT functionals have been made. The location of the $\mathrm{Cd} 4 d$ states which have a significant effect on the theoretical predictions was not reproduced by either LDA or PBE-GGA. However, LDA + U applied using a theoretically calculated $\mathrm{U}$ value of $2.34 \mathrm{eV}$ was very successful in predicting the location of these states. Experimentally, the VB shows a two peak structure which is correctly reproduced by all the DFT functionals. However, the VB width is underestimated in each case, and the application of LDA + U does not improve this. The photon energy dependence of the VB-PES was measured and compared to the theoretical predictions. All the DFT functionals qualitatively reproduced the observed behavior, with LDA + U again being the most accurate. The data obtained with the lowest photon energy indicated the orbital character of the VB was not completely reproduced by the DFT calculations. Additionally the center of the VB shows a photon energy dependence which wasn't adequately replicated by any of the DFT functionals. This was assigned to $\mathrm{Cd} 5 p$ character and suggests it is underestimated by the DFT approaches. The trends observed with photon energy indicate that the $\mathrm{O} 2 p$ photoionization cross section decreases too rapidly with increasing photon energy when compared with the experimental data. This trend is in agreement with observations made in previous studies [34-36].

The change in VB shape with photon energy highlights the potential for this kind of approach in understanding orbital character present in the VB, and by varying the polarization even more detailed information can be obtained by allowing certain orbital character to be suppressed. In order to exploit this information further, improvements in the photoionization cross sections at the high photon energies relevant for HAXPES or one-step photoemission calculations will be required. All the DFT functionals applied here significantly underestimate the $\mathrm{CdO}$ band gap despite their successes in predicting the VB shape and $\mathrm{Cd} 4 d$ position. This clearly illustrates the need for more sophisticated theoretical approaches such as hybrid functionals and GW quasiparticle methods, when investigating these materials. It is anticipated that these experimental results will be of use when assessing the successes of these approaches.

\section{ACKNOWLEDGMENTS}

We are grateful to P. K. Thakur (Diamond Light Source, UK) for assistance with SR-PES measurements, and acknowledge the EPSRC for the award of a DTA studentship (J.J.M.). The work undertaken at the Universitat de Valéncia was supported by the Spanish Government under Project No. TEC2011-28076-C02-02, and Generalitat Valenciana under the projects Prometeo/2011-035 and ISIC/2012/008, Institute of Nanotechnologies for Clean Energies. The Science City Research Alliance (SCRA) and the European Research and Development Fund (ERDF) are acknowledged for capital equipment investment at Warwick. Diamond Light Source are thanked for awarding beamtime under the proposal SI 8441.
[1] K. M. Yu, M. A. Mayer, D. T. Speaks, H. He, R. Zhao, L. Hsu, S. S. Mao, E. E. Haller, and W. Walukiewicz, J. Appl. Phys. 111, 123505 (2012).
[2] T. J. Coutts, D. L. Young, X. Li, W. P. Mulligan, and X. Wu, J. Vac. Sci. Technol. A 18, 2646 (2000). 
[3] R. Chandiramouli and B. Jeyaprakash, Solid State Sci. 16, 102 (2013).

[4] R. Miloua, F. Miloua, A. Arbaoui, Z. Kebbab, and N. Benramdane, Solid State Commun. 144, 5 (2007).

[5] A. Schleife, C. Rödl, J. Furthmüller, and F. Bechstedt, New J. Phys. 13, 085012 (2011).

[6] P. D. C. King and T. D. Veal, J. Phys. Condens. Matter 23, 334214 (2011).

[7] L. Y. Lim, S. Lany, Y. J. Chang, E. Rotenberg, A. Zunger, and M. F. Toney, Phys. Rev. B 86, 235113 (2012).

[8] A. Schleife, F. Fuchs, J. Furthmüller, and F. Bechstedt, Phys. Rev. B 73, 245212 (2006).

[9] M. Burbano, D. O. Scanlon, and G. W. Watson, J. Am. Chem. Soc. 133, 15065 (2011).

[10] F. Labat, P. Baranek, C. Domain, C. Minot, and C. Adamo, J. Chem. Phys. 126, 154703 (2007).

[11] H. Dixit, D. Lamoen, and B. Partoens, J. Phys. Condens. Matter 25, 035501 (2013).

[12] A. Schleife, F. Fuchs, C. Rödl, J. Furthmüller, and F. Bechstedt, Phys. Status Solidi 246, 2150 (2009).

[13] V. I. Anisimov, J. Zaanen, and O. K. Andersen, Phys. Rev. B 44, 943 (1991).

[14] A. I. Liechtenstein, V. I. Anisimov, and J. Zaanen, Phys. Rev. B 52, R5467 (1995).

[15] V. I. Anisimov, F. Aryasetiawan, and A. I. Lichtenstein, J. Phys. Condens. Matter 9, 767 (1997).

[16] P. D. C. King, T. D. Veal, A. Schleife, J. Zúñiga-Pérez, B. Martel, P. H. Jefferson, F. Fuchs, V. Muñoz-Sanjosé, F. Bechstedt, and C. F. McConville, Phys. Rev. B 79, 205205 (2009).

[17] Y. Dou, R. G. Egdell, D. S. L. Law, N. M. Harrison, and B. G. Searle, J. Phys. Condens. Matter 10, 8447 (1998).

[18] J. J. Mudd, T. L. Lee, V. Muñoz-Sanjosé, J. Zúñiga-Pérez, D. Hesp, J. M. Kahk, D. J. Payne, R. G. Egdell, and C. F. McConville, Phys. Rev. B 89, 035203 (2014).

[19] M. Trzhaskovskaya, V. Nefedov, and V. Yarzhemsky, At. Data Nucl. Data Tables 77, 97 (2001).

[20] M. Trzhaskovskaya, V. Nikulin, V. Nefedov, and V. Yarzhemsky, At. Data Nucl. Data Tables 92, 245 (2006).

[21] J. Zuñiga Pérez, C. Munuera, C. Ocal, and V. Muñoz-Sanjosé, J. Cryst. Growth 271, 223 (2004).

[22] D. Singh and L. Nordström, Planewaves, Pseudopotentials, and the LAPW method, 2nd ed. (Springer, New York, 2005).
[23] "Elk FP-LAPW DFT Code," http://elk.sourceforge.net/.

[24] J. P. Perdew and A. Zunger, Phys. Rev. B 23, 5048 (1981).

[25] J. P. Perdew, K. Burke, and M. Ernzerhof, Phys. Rev. Lett. 77, 3865 (1996).

[26] A. Janotti, D. Segev, and C. G. Van de Walle, Phys. Rev. B 74, 045202 (2006).

[27] F. D. Murnaghan, Proc. Natl. Acad. Sci. USA 30, 244 (1944).

[28] S. K. Vasheghani Farahani, V. Munoz-Sanjose, J. Zuniga-Perez, C. F. McConville, and T. D. Veal, Appl. Phys. Lett. 102, 022102 (2013).

[29] I. N. Demchenko, J. D. Denlinger, M. Chernyshova, K. M. Yu, D. T. Speaks, P. Olalde-Velasco, O. Hemmers, W. Walukiewicz, A. Derkachova, and K. Lawniczak-Jablonska, Phys. Rev. B 82, 075107 (2010).

[30] J. W. Cooper, Phys. Rev. A 47, 1841 (1993).

[31] P. D. C. King, T. D. Veal, C. F. McConville, J. Zúñiga-Pérez, V. Muñoz-Sanjosé, M. Hopkinson, E. D. L. Rienks, M. F. Jensen, and P. Hofmann, Phys. Rev. Lett. 104, 256803 (2010).

[32] L. F. J. Piper, L. Colakerol, P. D. C. King, A. Schleife, J. Zúñiga-Pérez, P. A. Glans, T. Learmonth, A. Federov, T. D. Veal, F. Fuchs, V. Muñoz-Sanjosé, F. Bechstedt, C. F. McConville, and K. E. Smith, Phys. Rev. B 78, 165127 (2008).

[33] D. A. Shirley, Phys. Rev. B 5, 4709 (1972).

[34] D. J. Payne, R. G. Egdell, G. Paolicelli, F. Offi, G. Panaccione, P. Lacovig, G. Monaco, G. Vanko, A. Walsh, G. W. Watson, J. Guo, G. Beamson, P.-A. Glans, T. Learmonth, and K. E. Smith, Phys. Rev. B 75, 153102 (2007).

[35] D. J. Payne, G. Paolicelli, F. Offi, G. Panaccione, P. Lacovig, G. Beamson, A. Fondacaro, G. Monaco, G. Vanko, and R. G. Egdell, J. Electron Spectrosc. Relat. Phenom. 169, 26 (2009).

[36] C. Körber, V. Krishnakumar, A. Klein, G. Panaccione, P. Torelli, A. Walsh, J. L. F. Da Silva, S.-H. Wei, R. G. Egdell, and D. J. Payne, Phys. Rev. B 81, 165207 (2010).

[37] J. Scofield, Lawrence Livermore Lab. Report No. UCRL-51326, 1973 (unpublished).

[38] J. Yeh and I. Lindau, At. Data Nucl. Data Tables 32, 1 (1985).

[39] W. Drube, T. M. Grehk, S. Thieß, G. B. Pradhan, H. R. Varma, P. C. Deshmukh, and S. T. Manson, J. Phys. B: At. Mol. Opt. Phys. 46, 245006 (2013). 